Para enlazar con este artículo / To link to this article:

http://dx.doi.org/10.14198/fem.2019.33.09

Para citar este artículo / To cite this article:

Botello-Hermosa, Alicia, García-Jiménez, María, Santana-Berlanga, Nicia del Rocio y Ruiz-Ferrón, Cecilia. «Diseño y validación de un instrumento para medir los conocimientos y actitudes de las mujeres jóvenes ante la menstruación: escala Metcon (Botello-Hermosa 2018)». En Feminismo/s, 33 (junio 2019): 225-247. DOI: $10.14198 / \mathrm{fem} .2019 .33 .09$

\title{
DISEÑO Y VALIDACIÓN DE UN INSTRUMENTO PARA MEDIR LOS CONOCIMIENTOS Y ACTITUDES DE LAS MUJERES JÓVENES ANTE LA MENSTRUACIÓN: ESCALA METCON (BOTELLO-HERMOSA 2018)
}

\author{
DESIGN AND VALIDATION OF A QUESTIONNAIRE TO \\ MEASURE KNOWLEDGE AND ATTITUDES TOWARDS \\ MENSTRUATION IN YOUNG PEOPLE: ESCALA METCON \\ (BOTELLO-HERMOSA 2018)
}

Alicia Botello-HeRmosA Universidad de Sevilla abotello@us.es Orcid 0000-0001-6337-0971

María GARCÍA-JIMÉNEZ

Universidad de Sevilla mgarciaj@us.es Orcid 0000-0002-4751-061X

Nicia del Rocio SANTANA-BERLANGA

Universidad de Sevilla nicia.santana@hotmail.com Orcid 0000-0002-7650-3101

Cecilia RUIZ-FERRÓN

Universidad de Sevilla ceciliaruizferron@gmail.com Orcid 0000-0002-2821-8103

Los contenidos de la revista se publican bajo una licencia de Creative Commons Reconocimiento 4.0 Internacional (CC BY 4.0)

Feminismo/s 33, junio 2019, pp. 225-247 
Alicia Botello-Hermosa, María García-JimÉneZ,

Nicia DEl Rocio SANTANA-BERLANGA y CECILIA RUIZ-FERRÓN

Diseño y validación de un instrumento para medir los conocimientos y actitudes de las mujeres jóvenes ante la menstruación: escala Metcon (Botello-Hermosa 2018)

\title{
Resumen
}

Objetivo: diseñar y validar un instrumento específico para valorar los mitos, estereotipos, tabúes y conocimientos sobre la menstruación. Diseño: estudio instrumental en el que se diseñó y validó un cuestionario. Emplazamiento: Facultad de Enfermería, Fisioterapia y Podología de la Universidad de Sevilla. Participantes: 182 estudiantes. Intervenciones: índice de Osterlind, alfa de Cronbach, prueba KMO (Kaiser-Meyer-Olkin), test de esfericidad de Bartlett y método Varimax con normalización Kaiser. Mediciones principales: mitos, estereotipos, tabúes y conocimientos sobre la menstruación. Resultados: Se han realizado tres análisis factoriales, obteniendo un valor de convergencia general que explica el 67.978 \% de la varianza total y fiabilidad global de .72422 ítems con 22 ítems y una validez de constructo que demuestra la existencia de 4 dimensiones con .653 en la prueba KMO y 777.53 en el índice de esfericidad de Barlett ( $\mathrm{p}<.001)$ y todos los ítems obtuvieron comunalidades adecuadas $(>.50)$. Conclusiones: se ha obtenido una herramienta útil y válida para evaluar los mitos, estereotipos, tabúes y conocimientos sobre la menstruación que permita un manejo adecuado de la misma en consultas de Atención Primaria y/o en el entorno escolar.

Palabras clave: menstruación; experiencias menstruación; tabú; creencias populares; salud de las mujeres; educación.

\begin{abstract}
Objective: Design and validate a specific instrument to assess myths, stereotypes, taboos, and knowledge about menstruation. Design: Instrumental study in which a questionnaire was designed and validated. Location: Faculty of Nursing, Physiotherapy and Podiatry of the University of Seville. Participants: 182 students. Interventions: Osterlind index, Cronbach's alpha, KMO test (Kaiser-Meyer-Olkin), Bartlett's sphericity test and Varimax method with Kaiser normalization. Main measurements: Myths, stereotypes, taboo and knowledge about menstruation. Results: Three factorial analyzes were performed, obtaining a general convergence value that explains $67.978 \%$ of the total variance and global reliability of .72422 items with 22 items and a construct validity that demonstrates the existence of 4 dimensions with.653 in the KMO test and 777.53 in the Barlett sphericity index $(\mathrm{p}<.001)$ and all the items obtained adequate communalities (>.50). Conclusions: A useful and valid tool has been obtained to evaluate the myths, taboo stereotypes and knowledge about menstruation that allows an adequate management of it in primary care consultations and / or in the school environment.
\end{abstract}

Keywords: Menstruation; Menstruation experiences; Taboo; Popular beliefs; Women's health; Education.

Feminismo/s 33, junio 2019, pp. 225-247 


\section{INTRODUCCION}

La menstruación y el ciclo menstrual tienen en la mayoría de las sociedades connotaciones de suciedad e impureza, y en muchas ocasiones, además, se asocian a un símbolo de debilidad (Sau 49). Las creencias y actitudes colectivas hacia el ciclo menstrual no solo parecen intervenir en lo que experimentan las mujeres, sino que se emplean como explicaciones para la conducta femenina, afectando a la manera en que una mujer menstruante es vista y aceptada en los planos públicos y privados, y en cómo se le asignan actividades permitidas y no permitidas según su sexo (Feijóo Tituana 12).

De hecho, al asociarse la menstruación a suciedad e impureza se articulan prohibiciones en las mujeres menstruantes, como la prohibición de ir a bodegas de vino, de participar en elaboración de embutidos, de recoger frutos..., mitos que se han ido transmitiendo de forma oral de generación en generación, algunos incluso desde la época romana (basados en parte en las teorías hipocráticas de los humores), y han llegado hasta nuestros días articulando miedos y prohibiciones (Botello-Hermosa 254).

En este sentido, la menstruación y todo lo relacionado con este proceso fisiológico, tiene cierta carga implícita de violencia simbólica, definida como relación social donde el «dominador» ejerce un modo de violencia indirecta y no físicamente directa en contra de los «dominados», los cuales no la evidencian o son inconscientes de dichas prácticas en su contra, por lo cual son «cómplices de la dominación a la que están sometidos» (Bourdieu 3).

La violencia simbólica implícita en la menstruación también se visibiliza paradójicamente en la forma de ocultarlo; el tabú o silencio de mujeres y hombres sobre el tema (Valls-Llobet 134). Los comentarios negativos por parte de la sociedad, hombres y mujeres, relacionados con impureza y suciedad en torno a la menstruación canalizan sentimientos de inseguridad y vergüenza que las llevan a ellas a guardar silencio (Newton), convirtiéndolas en cómplices de su propia victimización (Bourdieu 3).

Así mismo, las ideas que se transmiten en el entorno familiar a los hijos/ as intervienen en sus ideas acerca de la menstruación. Es así que muchas veces, las madres y padres son actores inconscientes en el proceso de violencia simbólica dentro de la menstruación por ocultarlo a los hombres de la familia o hacer que sus hijas vivan la menstruación con vergüenza 
hacia ellos (Feijóo Tituana 14). En nuestro entorno, por ejemplo, se utilizan eufemismos para referirse a la menstruación como «la regla», «el periodo», «estar mala», «ya llegó mi primo» o «ya se ha puesto los pendientes rojos», entre otros (Botello-Hermosa 267). En todo el mundo se calcula que pueden existir 60 billones de eufemismos relacionados con el ciclo menstrual (Stein y Kim 142).

En este sentido, es importante destacar que el Convenio de Estambul ratificado por España y publicado en el BOE el 6 de junio de 2014, reconoce que la naturaleza estructural de la violencia contra las mujeres está basada en el género, por lo que en el Capítulo III: Prevención, el artículo 12 «Obligaciones generales» indica que es necesario promover cambios en los modos de comportamientos socioculturales de mujeres y hombres con vistas a erradicar los prejuicios, costumbres, tradiciones, y cualquier otra práctica basada en la inferioridad de la mujer o en un papel estereotipado de mujeres y hombres. En el artículo 14, «Educación», vuelve a resaltar la importancia de realizar acciones necesarias para incluir papeles no estereotipados.

De acuerdo con Martínez, Parera y Rius, para un apropiado manejo de la menstruación es necesario conocer cómo la vive la mujer y cómo le afecta y limita, factores influenciados por el entorno sociocultural. La información acerca de estos aspectos en la mujer española es escasa (71).

Con respecto a los instrumentos para estudiar la menstruación desde un punto de vista social y cultural, tras la búsqueda bibliográfica es importante señalar que no existen instrumentos validados con suficiente fiabilidad. Destaca el Menstrual Attitude Questionnaire (MAQ) (Brooks-Gunn y Ruble) que fue construido y validado en Nueva York. Posteriormente fue validado en Turquía (Firat et al.). El objetivo del MAQ era analizar las actitudes frente a la menstruación y sus efectos. No estudia las creencias populares, ni los estereotipos de género, ni los conocimientos.

Por todo ello, el objetivo de esta investigación fue diseñar y validar un instrumento específico para analizar los mitos, estereotipos de género, tabúes y conocimientos que sobre la menstruación tienen las jóvenes españolas: Escala METCON (Botello-Hermosa 2018). 


\section{MÉTODO}

El diseño del cuestionario se realizó a partir de la revisión bibliográfica y del análisis de contenido de 24 entrevistas en profundidad y 3 grupos de discusión realizados como parte de la tesis doctoral elaborada con investigación cualitativa, Aproximación a las creencias populares sobre los ciclos vitales femeninos desde la perspectiva de género (Botello-Hermosa). Las categorías encontradas configuraron las dimensiones básicas de este instrumento, de manera que se tomaron como ítems adaptaciones de fragmentos de las propias mujeres. Para la realización de la escala se exploró la validez de aspecto y de contenido mediante la técnica de Osterlind (1989).

Diseño del cuestionario:

\subsection{Definición de dimensiones y atributos y redacción de ítems}

El instrumento tiene cuatro dimensiones:

1. La menstruación como tema tabú. Esta dimensión pretende medir si la menstruación es un tema tabú y en tal caso, la utilización de herramientas como el lenguaje metafórico para referirse a tal hecho. Además, pretende determinar los entornos donde existe tabú sobre la menstruación: el entorno familiar, el grupo de iguales o el entorno escolar.

2. Estereotipos de género relacionados con la menstruación. Se pretende conocer estereotipos de género (culturales) relacionados con la menstruación. Se analizan estereotipos sobre la menarquía (comienzo de la etapa fértil) y la menopausia (fin de la menstruación).

3. Creencias falsas y mitos sobre la menstruación. Tiene cuatro subdimensiones directamente relacionadas: mitos, creencias falsas, miedos y prohibiciones relacionados con la menstruación.

4. Conocimientos sobre la menstruación. En esta dimensión se pretende analizar los conocimientos sobre el funcionamiento biológico y fisiológico del ciclo menstrual y de la menstruación que refieren tener, y si ellas los consideran suficientes.

Para la redacción de los enunciados que finalmente compondrían el instrumento, se intentó evitar el uso de oraciones confusas, como varias negaciones dentro de un mismo ítem, y utilizar términos coloquiales para facilitar su 
Alicia Botello-Hermosa, María García-Jiménez,

Nicia del Rocio SANTANA-BERlanga y CECILIA Ruiz-FERRón

Diseño y validación de un instrumento para medir los conocimientos y actitudes de

las mujeres jóvenes ante la menstruación: escala Metcon (Botello-Hermosa 2018)

comprensión. Se redactó un total de 69 ítems iniciales. Los ítems adoptaron un formato de respuesta de escala tipo Likert de 0 a 6 , en la que « 0 » significa totalmente en desacuerdo o nunca, «1» muy en desacuerdo o muy pocas veces, « 2 » poco en desacuerdo o pocas veces, « 3 » algo de acuerdo o alguna vez, «4» bastante de acuerdo o bastantes veces, « $5 »$ muy de acuerdo o muchas veces $\mathrm{y} « 6 »$ No sabe/ No contesta.

\subsection{Validación de aspecto y de contenido}

Para evaluar la calidad descriptiva de los ítems, así como una forma de asegurar la validez interna de los mismos y la validez de contenido, se calculó el índice propuesto por Osterlind en base al juicio de cinco expertas. El perfil de las expertas consultadas fue: Medicina preventiva y Salud pública, Psicología social, Teoría e Historia de la Educación y Pedagogía Social, Enfermería y Promoción de la Salud, y Estudios de las Mujeres y de Género. Las expertas participaron en la evaluación de la calidad de los ítems, en relación al objetivo o dimensión que cada uno de ellos pretendía medir. La tarea de cada experta consistía en evaluar, de forma independiente, cada uno de los ítems según el grado (alto, medio o bajo) en que se relacionaban con la dimensión para la que se había creado. La tabla 1 muestra los ítems con un índice de Osterlind superior a .5.

Tabla 1. Ítems por dimensiones e índice de Osterlind (I)

\begin{tabular}{|l|c|}
\hline \multicolumn{1}{|c|}{ Ítems de la dimensión 1. «La menstruación como tema tabú» } & I \\
\hline 1. En casa la menstruación era un tema tabú. & .80 \\
$\begin{array}{l}\text { 2. Hubo personas a mi alrededor (familiares, amigas/os, en el colegio o } \\
\text { instituto) que me dijeron qué era la menstruación y me dieron información } \\
\text { sobre ello. }\end{array}$ & .60 \\
3. En el colegio/instituto la menstruación era un tema más tabú que en casa. & .80 \\
$\begin{array}{l}\text { 4. Entre amigas, primas o hermanas, la menstruación era un tema menos tabú } \\
\text { que en casa. }\end{array}$ & 1.00 \\
$\begin{array}{l}\text { 5. Entre amigas, primas o hermanas, la menstruación era un tema menos tabú } \\
\text { que en el colegio/instituto. }\end{array}$ & 1.00 \\
$\begin{array}{l}\text { 8. Cuando por primera vez tuve la menstruación sabía lo que era con } \\
\text { seguridad. }\end{array}$ & .60 \\
9. Me asusté cuando vi por primera vez la sangre menstrual. & .60 \\
\hline
\end{tabular}

Feminismo/s 33, junio 2019, pp. 225-247 
Alicia Botello-Hermosa, María García-JimÉneZ,

Nicia del Rocio SANTANA-Berlanga y Cecilia Ruiz-FERrón

Diseño y validación de un instrumento para medir los conocimientos y actitudes de las mujeres jóvenes ante la menstruación: escala Metcon (Botello-Hermosa 2018)

\begin{tabular}{|c|c|}
\hline $\begin{array}{l}\text { 1. Con la primera menstruación me dijeron expresiones como «Ya te has } \\
\text { hecho mujer», «ya eres mujercita»... }\end{array}$ & 1,00 \\
\hline 2. La menarquía supone el paso de niña a mujer. & 1.00 \\
\hline $\begin{array}{l}\text { 3. No sentí con la menarquia (primera menstruación) la sensación de haber } \\
\text { crecido de repente. }\end{array}$ & .80 \\
\hline $\begin{array}{l}\text { 4. Con la menstruación me dijeron que ya no podía jugar con niños o que } \\
\text { tenía que tener cuidado con ellos (se refiere a los niños/hombres). }\end{array}$ & .80 \\
\hline $\begin{array}{l}\text { 5. Mi madre o algún familiar avisaba (por teléfono, personalmente...) como } \\
\text { motivo de alegría al resto de familiares o vecinos que ¡ya me había venido la } \\
\text { regla! o que iya era mujer! }\end{array}$ & .60 \\
\hline $\begin{array}{l}\text { 8. No le dije a nadie cuando me llegó la menarquia (primera menstruación) } \\
\text { por vergüenza. }\end{array}$ & .60 \\
\hline 12. Durante la menstruación se está mala o enferma. & 1.00 \\
\hline $\begin{array}{l}\text { 14. La menopausia supone el envejecimiento de la mujer porque ya no tiene } \\
\text { menstruación. }\end{array}$ & 1.00 \\
\hline 15. Durante la menopausia las mujeres ya no sirven para nada. & .80 \\
\hline 16. Al llegar a la menopausia se pierde el atractivo sexual. & .80 \\
\hline $\begin{array}{l}\text { 17. Durante la menopausia se requiere tratamiento médico (ansiedad, } \\
\text { depresión, sofocos...). }\end{array}$ & .60 \\
\hline \multicolumn{2}{|l|}{ Ítems de la dimensión 3. «Creencias falsas y mitos sobre la menstruación» } \\
\hline $\begin{array}{l}\text { 1. Lavarse la cabeza durante la menstruación puede ser peligroso para la } \\
\text { salud. }\end{array}$ & 1.00 \\
\hline $\begin{array}{l}\text { 2. Durante la menstruación no tengo más riesgo de ataque de animales por el } \\
\text { olor como perros, culebras... }\end{array}$ & .80 \\
\hline $\begin{array}{l}\text { 3. Si preparo alimentos durante la menstruación como mayonesa, se estropea } \\
\text { o corta con facilidad. }\end{array}$ & 1.00 \\
\hline $\begin{array}{l}\text { 4. No hay relación entre tocar las flores o plantas durante la menstruación y } \\
\text { que se estropeen o sequen. }\end{array}$ & .60 \\
\hline 5. Durante la menstruación no hay que bañarse ni en el mar ni en las piscinas. & 1.00 \\
\hline $\begin{array}{l}\text { 6. La sangre menstrual es tóxica, por lo que puede tener efectos perjudiciales } \\
\text { en las cosechas. }\end{array}$ & .80 \\
\hline $\begin{array}{l}\text { 7. Durante la menstruación no hay que ducharse porque se corta y es } \\
\text { peligroso para la salud. }\end{array}$ & 1.00 \\
\hline
\end{tabular}

Feminismo/s 33, junio 2019, pp. 225-247 
Alicia Botello-Hermosa, María García-JimÉneZ,

Nicia del Rocio SANTANA-Berlanga y Cecilia Ruiz-FERrón

Diseño y validación de un instrumento para medir los conocimientos y actitudes de las mujeres jóvenes ante la menstruación: escala Metcon (Botello-Hermosa 2018)

8. La sangre menstrual es un cúmulo de suciedad y desechos.

9. La sangre menstrual tiene un olor desagradable a descomposición.

10. La sangre menstrual es incompatible con el agua.

11. Durante la menstruación no se deben tener relaciones sexuales.

14. Durante la menstruación se está impura.

1.00

15. Actúo de diferente forma (relacionado con mi religión) cuando tengo la menstruación.

16. Existen prohibiciones específicas que yo sigo durante los días que tengo la menstruación.

Ítems de la dimensión 4. «Conocimientos sobre la menstruación»

1. No conozco las diferencias entre ciclo menstrual y menstruación.

2. Identifico las diferentes etapas del ciclo menstrual.

3. No sabría reconocer los indicadores de un ciclo menstrual alterado.

4.Conozco en qué momento del ciclo menstrual soy más fértil .60

5. Conozco mi ciclo menstrual y lo que ocurre en mi cuerpo.

6. Debería recibir más información sobre el ciclo menstrual.

1.00

7. Debería recibir más información sobre la menstruación. .60

8. No conozco bien el funcionamiento fisiológico de mis ciclos. .60

9. Conocer mejor mi ciclo menstrual me ayudaría a prevenir embarazos no deseados junto con el uso de anticonceptivos.

10. Conozco cómo afectan los anticonceptivos hormonales al ciclo menstrual.

11. No identifico las hormonas que regulan mi ciclo menstrual.

12. La píldora del día después produce una menstruación.

13. Tener relaciones sexuales durante la menstruación dificulta posibles embarazos.

14. La menstruación es un proceso fisiológico absolutamente normal por lo que no se tienen que tener cuidados específicos.

15. Tengo menstruaciones dolorosas por lo que en algún momento me automedico.

16. Conozco algún medicamento específico para la dismenorrea (dolor en la menstruación).

17. Conozco las características de mi ciclo menstrual. 
Diseño y validación de un instrumento para medir los conocimientos y actitudes de las mujeres jóvenes ante la menstruación: escala Metcon (Botello-Hermosa 2018)

18. Considero que la formación que recibí en casa sobre la menstruación fue correcta y adecuada.

21. Conozco el proceso fisiológico de la menopausia y las hormonas implicadas para ello.

Del total de 69 ítems iniciales, tras el juicio de las expertas, se eliminaron aquellos ítems que obtuvieron un índice de Osterlind inferior a .5. Se eliminaron 19 ítems, quedando un total de 50. Hay que indicar que, finalizado el panel de expertas con evaluaciones de tipo cuantitativo, se tuvieron en consideración las sugerencias para que algunos ítems fueran ligeramente modificados y adaptados en su redacción, así como que cambiaran de dimensión: el ítem 8 de la dimensión 2 pasó a la dimensión 1.

\subsection{Prueba piloto}

Se realizó una prueba piloto con 62 estudiantes universitarias de $2 .^{\circ}$ curso del Grado en Enfermería de la Universidad de Sevilla durante el mes de noviembre de 2016. El objetivo fue identificar preguntas ambiguas o confusas, posibles errores y conflictos de comprensión. Al finalizar, los hombres que participaron indicaron que no podían contestar a muchas preguntas ya que estaban basadas en experiencias propias sobre el ciclo menstrual y que respondían en función a las experiencias conocidas en su entorno de madres, amigas o hermanas, lo cual descartó la posibilidad de ser un instrumento dirigido a hombres. Aparte de esto, tras el pilotaje, no se modificó, añadió ni eliminó ningún ítem.

\subsection{Estudio de campo}

Del 16 al 22 de diciembre de 2016 se realizó el estudio de campo con la versión preliminar (50 ítems a 120 estudiantes mujeres (sin considerar criterios de exclusión para la muestra) del Grado de Enfermería de la Facultad de Enfermería, Fisioterapia y Podología. La edad media fue de 20.5 años (DT=4.05). Pertenecía a medio rural el $47.5 \%$, y a medio urbano el $52.5 \%$ de las participantes. La participación fue voluntaria y anónima. El cuestionario fue autoadministrado y el tiempo necesario para responderlo osciló entre 
8 y 12 minutos. Los ítems se presentaron aleatorizados y con un pequeño encabezado con instrucciones básicas sobre cómo responder.

\subsection{Consideraciones éticas}

Este Proyecto de Investigación Biomédica cuenta con la aprobación y el dictamen favorable por parte del Comité Coordinador de Ética de la Investigación Biomédica de Andalucía. Así mismo, se siguió la Declaración de Helsinki (última revisión 2013) para la investigación con seres humanos. Todas las participantes rellenaron un consentimiento informado previamente a hacer el cuestionario.

\subsection{Análisis estadístico}

La fiabilidad del instrumento se determinó mediante la prueba alfa de Cronbach (1951), teniendo en cuenta que los valores de alfa relevantes eran los superiores a.70 y la validez de constructo a través del análisis factorial exploratorio por componentes principales, administrado a las cuatro dimensiones. Esta técnica se aplicó tras haber descartado previamente, mediante la prueba KMO (Kaiser-Meyer-Olkin) y el test de esfericidad de Bartlett, que las correlaciones entre los ítems constituyesen una matriz de identidad, lo que desaconsejaría totalmente el uso de análisis factoriales. Para la rotación de los componentes, dada la existencia de correlaciones entre los factores, se utilizó el método Varimax con normalización Kaiser.

Los análisis estadísticos se llevaron a cabo mediante el paquete estadístico SPSS 22.0.

\section{RESULTADOS}

La versión final del instrumento METCON es una escala de 22 ítems compuesta por cuatro dimensiones que miden los mitos, estereotipos de género, tabúes y conocimientos sobre la menstruación. Los análisis mostraron una fiabilidad global de .724 y la validez de constructo demostró la existencia de cuatro dimensiones con un índice KMO de 653 y un índice de esfericidad de Barlett de 777.53, ambos estadísticamente significativos, con un valor $\mathrm{p}<.001$. Además, la matriz de correlaciones obtuvo un determinante igual a .001. 
Se realizaron tres análisis factoriales. Las complicaciones del análisis factorial común han contribuido al uso generalizado del análisis de componentes principales. La investigación empírica ha mostrado resultados similares. Tanto el análisis de componentes principales como los análisis factoriales comunes llegan a resultados esencialmente idénticos. El primer análisis de fiabilidad mostró que, eliminando 26 ítems que alcanzaron suficiente consistencia interna de forma individual, la consistencia interna de cada dimensión y la consistencia interna global del instrumento queda tal y como aparece en la tabla 2.

Tabla 2. Alfa de Cronbach de cada dimensión y total tras primera depuración de ítems

\begin{tabular}{|l|c|c|}
\hline & $\begin{array}{c}\text { Alfa de } \\
\text { Cronbach }\end{array}$ & $\begin{array}{c}\text { N } \\
\text { ítems }\end{array}$ \\
\hline Dimensión 1. La menstruación como tema tabú. & .53 & 7 \\
Dimensión 2. Estereotipos de género relacionados con la & .64 & 5 \\
menstruación. & .70 & 9 \\
Dimensión 3. Creencias falsas y mitos sobre la menstruación. & .84 & 3 \\
Dimensión 4. Conocimientos sobre la menstruación. & .74 & 24 \\
\hline METCOM global & & \\
\hline
\end{tabular}

La eliminación de estos ítems permitió que el alfa de Cronbach pasara de.58 con 50 ítems, a.74 con sólo 24 ítems.

El análisis factorial exploratorio tras el primer análisis de fiabilidad mostró que el ítem 1 del objetivo 2 («Estereotipos de género relacionados con la menstruación») obtuvo una comunalidad de.37, lo que hizo necesario eliminar este ítem y realizar un segundo análisis factorial. De nuevo, el ítem 8 de la dimensión 1 ( «La menstruación como tema tabú») no tenía carga suficiente en ningún factor. Esto llevó a la realización de un tercer análisis factorial tras eliminar también éste ítem. Este tercer análisis factorial, con 22 ítems, obtuvo.653 en la prueba KMO y 777.53 en el índice de esfericidad de Barlett $(\mathrm{p}<.001)$ y todos los ítems obtuvieron comunalidades adecuadas (>.50) (Tabla 3). 
Alicia Botello-Hermosa, María García-JimÉneZ,

Nicia del Rocio SANTANA-BERlanga y CECILIA Ruiz-FERRón

Diseño y validación de un instrumento para medir los conocimientos y actitudes de

las mujeres jóvenes ante la menstruación: escala Metcon (Botello-Hermosa 2018)

Tabla 3. Comunalidades de cada ítem

\begin{tabular}{|c|c|c|}
\hline \multicolumn{3}{|l|}{ Comunalidades } \\
\hline & Inicial & Extracción \\
\hline $\begin{array}{l}\text { 1.2. Hubo personas a mi alrededor (familiares, amigas/os, en el } \\
\text { colegio o instituto) que me dijeron qué era la menstruación. }\end{array}$ & 1,000 & 693 \\
\hline $\begin{array}{l}\text { 1.3. En el colegio/instituto la menstruación era un tema más } \\
\text { tabú que en casa. }\end{array}$ & 1,000 & 602 \\
\hline $\begin{array}{l}\text { 1.4. Entre amigas, primas o hermanas, la menstruación era un } \\
\text { tema menos tabú que en casa. }\end{array}$ & 1,000 & ,718 \\
\hline $\begin{array}{l}\text { 1.5. Entre amigas, primas o hermanas, la menstruación era un } \\
\text { tema menos tabú que en el colegio/instituto. }\end{array}$ & 1,000 & ,743 \\
\hline $\begin{array}{l}\text { 1.6. Cuando por primera vez tuve la menstruación, sabía lo que } \\
\text { era y me sentí segura. }\end{array}$ & 1,000 & ,706 \\
\hline 1.7. Me asusté cuando vi por primera vez la sangre menstrual. & 1,000 & 694 \\
\hline 2.9. Al llegar a la menopausia se pierde el atractivo sexual. & 1,000 &, 660 \\
\hline $\begin{array}{l}\text { 2.10. Durante la menopausia se requiere tratamiento médico } \\
\text { (ansiedad, depresión, sofocos...). }\end{array}$ & 1,000 & ,666 \\
\hline 2.6. Durante la menstruación se está mala o enferma. & 1,000 & 666 \\
\hline $\begin{array}{l}\text { 2.7. La menopausia supone el envejecimiento de la mujer } \\
\text { porque ya no tiene menstruación. }\end{array}$ & 1,000 &, 576 \\
\hline $\begin{array}{l}\text { 3.1. Lavarse la cabeza durante la menstruación puede ser } \\
\text { peligroso para la salud. }\end{array}$ & 1,000 &, 567 \\
\hline $\begin{array}{l}\text { 3.3. Si preparo alimentos como la mayonesa durante la } \\
\text { menstruación se estropea o corta con facilidad. }\end{array}$ & 1,000 & ,749 \\
\hline $\begin{array}{l}\text { 3.5. Durante la menstruación no hay que bañarse ni en el mar ni } \\
\text { en las piscinas. }\end{array}$ & 1,000 & ,753 \\
\hline $\begin{array}{l}\text { 3.6. La sangre menstrual es tóxica, por lo que puede tener } \\
\text { efectos perjudiciales en alimentos o bebidas que se toquen } \\
\text { estando con la menstruación. }\end{array}$ & 1,000 & 661 \\
\hline $\begin{array}{l}\text { 3.7. Durante la menstruación no hay que ducharse porque se } \\
\text { corta y es peligroso para la salud. }\end{array}$ & 1,000 &, 571 \\
\hline 3.8. La sangre menstrual es un cúmulo de suciedad y desechos. & 1,000 &, 729 \\
\hline $\begin{array}{l}\text { 3.9. La sangre menstrual tiene un olor desagradable a } \\
\text { descomposición. }\end{array}$ & 1,000 & 609 \\
\hline 3.10. La sangre menstrual es incompatible con el agua. & 1,000 &, 721 \\
\hline $\begin{array}{l}\text { 3.11. Durante la menstruación no se deben tener relaciones } \\
\text { sexuales. }\end{array}$ & 1,000 &, 577 \\
\hline
\end{tabular}

Feminismo/s 33, junio 2019, pp. 225-247 
Alicia Botello-Hermosa, María García-Jiménez,

Nicia del Rocio SANTANA-Berlanga y CECILIA Ruiz-FERRón

Diseño y validación de un instrumento para medir los conocimientos y actitudes de las mujeres jóvenes ante la menstruación: escala Metcon (Botello-Hermosa 2018)

4.4. Conozco en qué momento del ciclo menstrual soy más fértil.

4.5. Conozco mi ciclo menstrual y lo que ocurre en mi cuerpo.

4.7. Necesito recibir más información sobre el ciclo menstrual.

\begin{tabular}{|l|l|}
1,000 &, 774 \\
1,000 &, 794 \\
1,000 &, 727
\end{tabular}

Método de extracción: análisis de componentes principales.

Todos los ítems obtuvieron una comunalidad superior a.57. Las comunalidades más altas corresponden al primer factor, «Conocimientos sobre la menstruación», que explica o aporta una mayor varianza al cuestionario final. Las comunalidades más bajas se aprecian para los ítems de la dimensión 3, «Creencias falsas y mitos sobre la menstruación» (Tabla 4).

Tabla 4. Rango de comunalidades para cada dimensión

\begin{tabular}{|l|c|}
\hline Dimensiones & Rango de extracción \\
\hline La menstruación como tema tabú. & $.69-.74$ \\
Estereotipos de género relacionados con la menstruación. & $.57-.67$ \\
Creencias falsas y mitos sobre la menstruación. & $.57-.75$ \\
Conocimientos sobre la menstruación. & $.73-.79$ \\
\hline
\end{tabular}

Por tanto, se realizaron tres análisis factoriales obteniendo finalmente un valor de convergencia general que explica el $67.98 \%$ de la varianza total y fiabilidad global de.724 (Tabla 5).

Tabla 5. Varianza total explicada

\begin{tabular}{|c|c|c|}
\hline Factor & \% de varianza explicada & \% acumulado de varianza explicada \\
\hline 1 & 13,22 & 13,22 \\
\hline 2 & 9,13 & 22,35 \\
\hline 3 & 8,50 & 30,85 \\
\hline 4 & 8,45 & 39,29 \\
\hline 5 & 7,95 & 47,25 \\
\hline 6 & 7,65 & 54,90 \\
\hline 7 & 6,81 & 61,71 \\
\hline 8 & 6,27 & 67,98 \\
\hline
\end{tabular}

Feminismo/s 33, junio 2019, pp. 225-247 
Alicia Botello-Hermosa, María García-JimÉneZ, Nicia del Rocio SANTANA-Berlanga y CeCilia Ruiz-FERrón

Diseño y validación de un instrumento para medir los conocimientos y actitudes de las mujeres jóvenes ante la menstruación: escala Metcon (Botello-Hermosa 2018)

En la matriz del componente rotado, se aprecian las 4 dimensiones con sus subdimensiones, que son en total los 9 factores que aparecen (Tabla 6).

\section{Tabla 6. Matriz del componente rotado}

\begin{tabular}{|c|c|c|c|c|c|c|c|c|}
\hline & \multicolumn{8}{|c|}{ Componente } \\
\hline & 1 & 2 & 3 & 4 & 5 & 6 & 7 & 8 \\
\hline 4.5. Conozco mi ciclo menstrual y lo que ocurre en mi cuerpo. &, 865 & & & & & & & \\
\hline 4.4. Conozco en qué momento del ciclo menstrual soy más fértil. &, 855 & & & & & & & \\
\hline 4.7. Necesito recibir más información sobre el ciclo menstrual. &, 802 & & & & & & & \\
\hline $\begin{array}{l}\text { 1.2. Hubo personas a mi alrededor (familiares, amigas/os, en el } \\
\text { colegio o instituto) que me dijeron qué era la menstruación }\end{array}$ &, 716 & & & & & & & \\
\hline 3.10. La sangre menstrual es incompatible con el agua. & &, 773 & & & & & & \\
\hline $\begin{array}{l}\text { 3.11. Durante la menstruación no se deben tener relaciones } \\
\text { sexuales. }\end{array}$ & & 643 & & & & & & \\
\hline $\begin{array}{l}\text { 3.7. Durante la menstruación no hay que ducharse porque se } \\
\text { corta y es peligroso para la salud. }\end{array}$ & &, 599 & & & & & & ,341 \\
\hline $\begin{array}{l}\text { 1.5. Entre amigas, primas o hermanas, la menstruación era un } \\
\text { tema menos tabú que en el colegio/instituto. }\end{array}$ & & & & & & & & \\
\hline $\begin{array}{l}\text { 1.4. Entre amigas, primas o hermanas, la menstruación era un } \\
\text { tema menos tabú que en casa. }\end{array}$ & & &, 798 & & & & & \\
\hline $\begin{array}{l}\text { 1.3. En el colegio/instituto la menstruación era un tema más tabú } \\
\text { que en casa. }\end{array}$ & & &, 671 & & & & & \\
\hline 2.9. Al llegar a la menopausia se pierde el atractivo sexual. & & & &, 720 & & & & \\
\hline 2.6. Durante la menstruación se está mala o enferma. & & & & ,688 & & & & \\
\hline $\begin{array}{l}\text { 2.7. La menopausia supone el envejecimiento de la mujer porque } \\
\text { ya no tiene menstruación. }\end{array}$ & & ,368 & &, 593 & & & & \\
\hline $\begin{array}{l}\text { 2.10. Durante la menopausia se requiere tratamiento médico } \\
\text { (ansiedad, depresión, sofocos...). }\end{array}$ & & & &, 555 & &, 375 & & \\
\hline $\begin{array}{l}\text { 3.6. La sangre menstrual es tóxica, por lo que puede tener efectos } \\
\text { perjudiciales en alimentos o bebidas que se toquen estando con } \\
\text { la menstruación. }\end{array}$ & & & & &, 757 & & & \\
\hline $\begin{array}{l}\text { 3.5. Durante la menstruación no hay que bañarse ni en el mar ni } \\
\text { en las piscinas. }\end{array}$ & & & & &, 705 & & &, 421 \\
\hline $\begin{array}{l}\text { 3.1. Lavarse la cabeza durante la menstruación puede ser } \\
\text { peligroso para la salud. }\end{array}$ & & & & &, 562 & & & \\
\hline 3.8. La sangre menstrual es un cúmulo de suciedad y desechos. & & & & & &, 790 & & \\
\hline $\begin{array}{l}\text { 3.9. La sangre menstrual tiene un olor desagradable a } \\
\text { descomposición. }\end{array}$ & & & & & &, 725 & & \\
\hline $\begin{array}{l}\text { 1.6. Cuando por primera vez tuve la menstruación sabía lo que } \\
\text { era y me sentí segura. }\end{array}$ & & & & & & & ,782 & \\
\hline 1.7. Me asusté cuando vi por primera vez la sangre menstrual. & & & & & & & ,772 & \\
\hline $\begin{array}{l}\text { 3.3. Si preparo alimentos como la mayonesa durante la } \\
\text { menstruación se estropea o corta con facilidad. }\end{array}$ & & & & & & & &, 830 \\
\hline
\end{tabular}

Nota: Método de extracción: análisis de componentes principales; Método de rotación: VARIMAX con normalización Kaiser.

Feminismo/s 33, junio 2019, pp. 225-247 
Alicia Botello-Hermosa, María García-Jiménez,

Nicia del Rocio SANTANA-BERlanga y CECILIA Ruiz-FERRón

Diseño y validación de un instrumento para medir los conocimientos y actitudes de las mujeres jóvenes ante la menstruación: escala Metcon (Botello-Hermosa 2018)

Se ha optado por la rotación VARIMAX para maximizar la suma de las varianzas de las cargas requeridas de la matriz de factores. No se ha optado por la rotación OBLIMIN, ya que la no ortogonalidad es específica a la muestra y no generalizable.

Se adjunta el gráfico de sedimentación, donde se indican los autovalores mayores de uno como criterio para la selección de factores.

Gráfico 1. Gráfico de sedimentación y número de componente

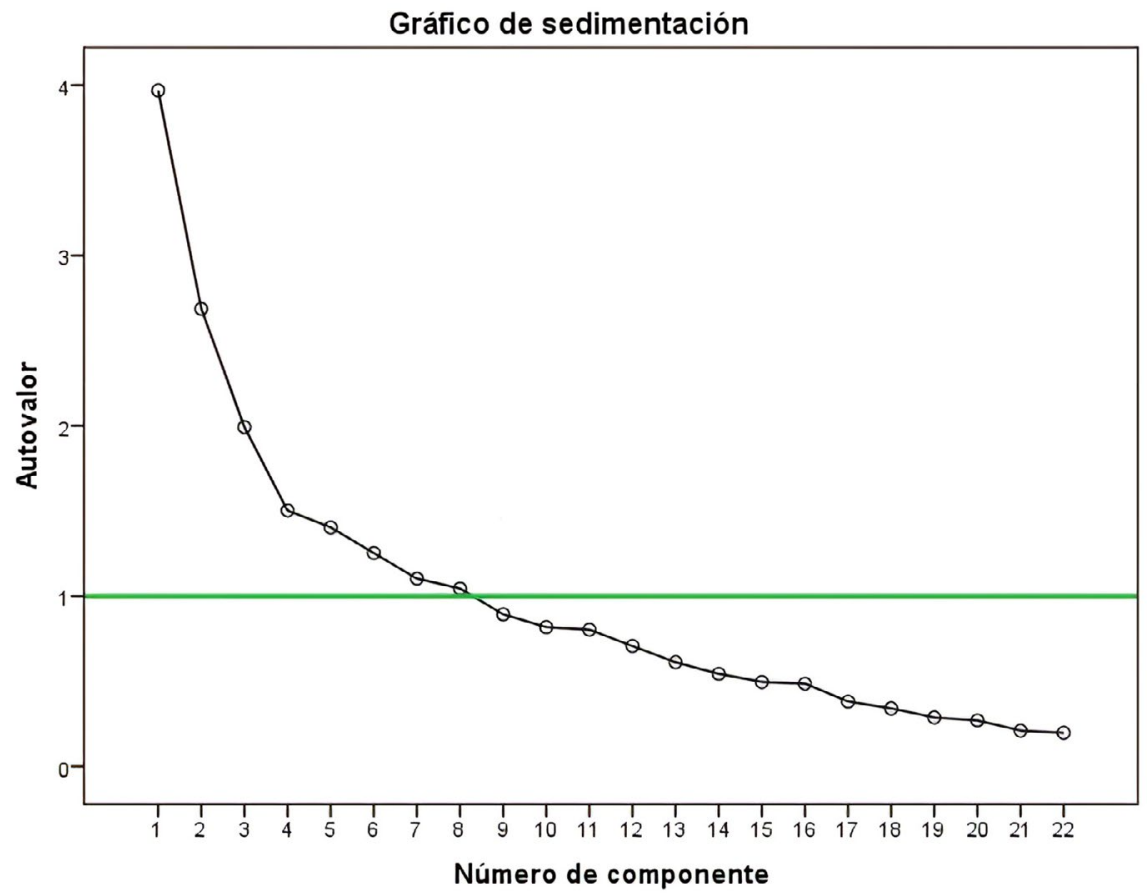

Feminismo/s 33, junio 2019, pp. 225-247 
Alicia Botello-Hermosa, María García-Jiménez, Nicia del Rocio SANTANA-BERlanga y CECILIA Ruiz-FERRón

Diseño y validación de un instrumento para medir los conocimientos y actitudes de las mujeres jóvenes ante la menstruación: escala Metcon (Botello-Hermosa 2018)

Por último, habiendo eliminado el ítem 1.8 del objetivo 1 y el ítem 2.1 del objetivo 2, como se señaló al principio, y añadiendo el hecho de que el análisis factorial mostró que el ítem 1.2 del objetivo 1 pertenecía en mayor medida a la dimensión 4, «Conocimientos sobre la menstruación», se calculó una vez más el alfa de Cronbach para comprobar la fiabilidad tras todas las modificaciones pertinentes (Tabla 7 ).

Tabla 7. Alfa de Cronbach de cada dimensión y total tras 1. a depuración de ítems

\begin{tabular}{|l|c|c|}
\hline & $\begin{array}{c}\text { Alfa de } \\
\text { Cronbach }\end{array}$ & $\begin{array}{c}\text { N } \\
\text { ítems }\end{array}$ \\
\hline Dimensión 1. La menstruación como un tema tabú. & .52 & 5 \\
$\begin{array}{l}\text { Dimensión 2. Estereotipos de género relacionados con la } \\
\text { menstruación. }\end{array}$ & .61 & 4 \\
Dimensión 3. Creencias falsas y mitos sobre la menstruación. & .70 & 9 \\
Dimensión 4. Conocimientos sobre la menstruación. & .84 & 4 \\
\hline METCOM global & .724 & 22 \\
\hline
\end{tabular}

A continuación, se muestra una tabla resumen del instrumento final METCON con la fiabilidad y el número de dimensiones con sus ítems. El total de varianza explicada es de 67,98\%.

Feminismo/s 33, junio 2019, pp. 225-247 
Alicia Botello-Hermosa, María García-Jiménez, Nicia del Rocio SANTANA-BERlanga y CECILIA Ruiz-FERRón

Diseño y validación de un instrumento para medir los conocimientos y actitudes de las mujeres jóvenes ante la menstruación: escala Metcon (Botello-Hermosa 2018)

Tabla 8. Características de la Escala METCON (Botello-Hermosa 2018)

\begin{tabular}{|c|c|c|c|c|}
\hline Dimensiones & Componentes & Ítems & $\begin{array}{c}\text { Alfa de Cronbach } \\
\text { (componentes) }\end{array}$ & $\begin{array}{c}\text { Alfa de } \\
\text { Cronbach } \\
\text { (dimensiones) }\end{array}$ \\
\hline \multirow{5}{*}{$\begin{array}{l}\text { La menstruación } \\
\text { como tema tabú }\end{array}$} & \multirow{3}{*}{$\begin{array}{l}\text { Entorno donde } \\
\text { existía el tabú }\end{array}$} & 1.5 & \multirow{3}{*}{0,662} & \multirow{5}{*}{0,518} \\
\hline & & 1.4 & & \\
\hline & & 1.3 & & \\
\hline & \multirow{2}{*}{$\begin{array}{l}\text { Experiencia } \\
\text { sobre el tabú }\end{array}$} & 1.6 & \multirow{2}{*}{0,538} & \\
\hline & & 1.7 & & \\
\hline \multirow{4}{*}{$\begin{array}{l}\text { Estereotipos de } \\
\text { Género }\end{array}$} & \multirow{4}{*}{$\begin{array}{l}\text { Estereotipos de } \\
\text { Género }\end{array}$} & 2.9 & \multirow{4}{*}{0,609} & \multirow{4}{*}{0,609} \\
\hline & & 2.6 & & \\
\hline & & 2.7 & & \\
\hline & & 2.10 & & \\
\hline \multirow{9}{*}{$\begin{array}{l}\text { Creencias falsas } \\
\text { y mitos sobre la } \\
\text { menstruación }\end{array}$} & \multirow{2}{*}{ Creencias falsas } & 3.8 & \multirow{2}{*}{0,612} & \multirow{9}{*}{0,700} \\
\hline & & 3.9 & & \\
\hline & \multirow{3}{*}{ Miedos } & 3.6 & \multirow{3}{*}{0,565} & \\
\hline & & 3.5 & & \\
\hline & & 3.1 & & \\
\hline & \multirow{3}{*}{ Prohibiciones } & 3.10 & \multirow{3}{*}{0,620} & \\
\hline & & 3.11 & & \\
\hline & & 3.7 & & \\
\hline & Mitos & 3.3 & & \\
\hline \multirow{4}{*}{$\begin{array}{l}\text { Conocimientos } \\
\text { sobre la } \\
\text { menstruación }\end{array}$} & \multirow{4}{*}{$\begin{array}{l}\text { Conocimientos } \\
\text { sobre la } \\
\text { menstruación }\end{array}$} & 4.5 & \multirow{4}{*}{0,840} & \multirow{4}{*}{0,840} \\
\hline & & 4.4 & & \\
\hline & & 4.7 & & \\
\hline & & 1.2 & & \\
\hline & & & & 0,724 (Global) \\
\hline
\end{tabular}

Feminismo/s 33, junio 2019, pp. 225-247 
Diseño y validación de un instrumento para medir los conocimientos y actitudes de las mujeres jóvenes ante la menstruación: escala Metcon (Botello-Hermosa 2018)

\section{DISCUSION}

\subsection{Dimensión 1. La menstruación como tema tabú}

\section{Componente 3. Entorno donde existía el tabú}

La menstruación sigue siendo un tema tabú entre adolescentes, en el sentido de que no se habla con naturalidad de ello principalmente con hombres de su entorno.

El tabú menstrual va unido además a conocimientos insuficientes que hacen que muchas niñas y adolescentes tengan que recurrir a su grupo de iguales por no obtener la información deseada ni en sus casas ni en los colegios o institutos. Sin embargo, mucha de esta información obtenida por iguales es errónea y estereotipada (Marván, Cortés-Iniestra, González 274; Larrañaga, Martín, Bacigalupe 114).

Esta falta de conocimientos está relacionada con que el tabú menstrual sigue estando presente en familias y contextos (Botello-Hermosa 183).

En este sentido, con respecto al ítem «Me asusté cuando vi por primera vez la sangre menstrual» el 40,9\% de las participantes contestó con de acuerdo y completamente de acuerdo.

Esta falta de conocimientos está relacionada con el tabú que todavía está presente, lo que refleja que el 65,2\% de las participantes contestó muy en desacuerdo o en desacuerdo con el ítem «Hubo personas a mi alrededor (familiares, amigas/os, en el colegio o instituto) que me dijeron que era la menstruación», lo que muestra que sigue siendo un tema tabú en algunas familias y contextos.

El tabú no aparece tanto entre iguales, como lo demuestra que el 39,2\% de las participantes estaba bastante de acuerdo y muy de acuerdo con el ítem "Entre amigas, primas o hermanas, la menstruación era un tema menos tabú que en casa» y el 23,3\% algo de acuerdo.

La familia y las amistades son la principal fuente de información sexual, seguida por la escuela y el centro de salud. Debido a las nuevas tecnologías, entre la población joven la escuela y los servicios de salud pierden importancia como fuentes de información y ganan en cambio las redes sociales e internet (Larrañaga, Martín, Bacigalupe 114). 
Alicia Botello-Hermosa, María García-Jiménez,

Diseño y validación de un instrumento para medir los conocimientos y actitudes de las mujeres jóvenes ante la menstruación: escala Metcon (Botello-Hermosa 2018)

\section{Componente 7. Experiencia sobre el tabú}

Esta falta de formación específica sobre la menstruación genera inseguridad en las adolescentes. Se subraya la idea de menstruación y violencia simbólica, provocando el tabú desconocimiento e inseguridad corporal, que las lleva a creer en la información informal que reciben por amigos o familiares de la misma edad (conocimientos entre iguales). Esta información no es siempre de la calidad y del rigor suficientes, por lo que se pueden seguir transmitiendo estereotipos e ideas erróneas asociadas a impureza e inferioridad; reproduciendo un papel estereotipado con respecto a la menstruación de mujeres y hombres (McClive 14).

\subsection{Dimensión 2. Estereotipos de género relacionados con la menstruación}

Componente 4. Estereotipos de género

A pesar de la atención dedicada desde la educación para la salud, en el ámbito de la salud sexual-reproductiva, a los cambios fisiológicos de la mujer (paradigma de feminidad: pubertad, menopausia), con objeto de configurar una concepción/percepción natural/con naturalidad de esos cambios fisiológicos, todavía existen estereotipos de género, destacando en este sentido los relacionados con la menopausia. La población más joven la concibe como signo de envejecimiento, por no tener menstruación (Botello- Hermosa y CasadoMejía, «Estereotipos»134).

\subsection{Dimensión 3, Creencias falsas y mitos sobre la menstruación}

Componente 6 Creencias falsas. Componente 5 Miedos. Componente 2. Prohibiciones. Componente 8. Mitos

Son factores influenciados por el entorno sociocultural que hay que tener en cuenta para una correcta educación sanitaria, ya que el conocer estas creencias falsas puede ayudar a erradicarlas (Goel y Mittal 50) (Botello-Hermosa y Casado-Mejía, «Miedos» 17).

Se cree que la idea de considerar la menstruación como un peligro comenzó en la prehistoria, por asociarse la contaminación con sangre menstrual con la atracción de animales hambrientos contra los cazadores. En Grecia (600 a.C.) fue considerada como una forma de eliminación de sustancias dañinas

Feminismo/s 33, junio 2019, pp. 225-247 
al cuerpo de la mujer, y en la medicina romana (siglo I d.C.) este concepto se mezcló con las ideas populares y mágicas sobre la sangre y se convierte en un elemento peligroso para las plantas animales y personas (Iglesia- Benavides 282).

Algunas de estas creencias han llegado hasta nuestros días mediante transmisión oral, sobre todo los miedos y prohibiciones relacionados con el uso del agua durante la menstruación por perjudicar gravemente a la salud, y el miedo a la toxicidad de la sangre menstrual. Nos planteamos si las falsas creencias milenarias se han visto erradicadas o si por el contrario, se han camuflado en «mitos» (Rus García 256)

Estas creencias falsas pueden afectar a las vivencias sobre la menstruación, por lo que para un manejo adecuado de la misma es importante conocerlas.

\subsection{Dimensión 4. Conocimientos sobre la menstruación}

\section{Componente 1. Conocimientos}

Con respecto al nivel de conocimientos de las encuestadas, se destaca que el $80,9 \%$ contestó (muy en desacuerdo o en desacuerdo) al ítem «Conozco mi ciclo menstrual y lo que ocurre en mi cuerpo», lo que demuestra la falta de conocimientos.

En este sentido, el 19,1\% de las participantes contestó que estaba entre de acuerdo y totalmente de acuerdo con el ítem «Necesito recibir más información sobre el ciclo menstrual»; y el 43,8 \% de las participantes contestó con de acuerdo y completamente de acuerdo con el ítem «Necesito recibir más información sobre la menstruación».

Existe un consenso creciente sobre la importancia de incidir en la educación sexual y reproductiva de una forma integral e integrada. Diferentes agentes sociales, formales y no formales, son responsables de cumplir esta función, entre los cuales destaca la escuela como escenario privilegiado para promover una sexualidad saludable desde edades tempranas (Barr et al. 10). Actualmente el sistema educativo y sanitario pierden relevancia como fuente de información, y medios como Internet cubren estas carencias, en ocasiones con contenidos poco objetivos y rigurosos (Larrañaga, Martín, Bacigalupe 114; Orringer y Gahagan 831). 
Es importante analizar qué nivel de conocimientos tienen las jóvenes con respecto al ciclo menstrual y a la menstruación, para incidir en su formación en el caso de que hiciera falta, en sus casas, en los colegios y en los Centros de Salud (Parera y Colomé 273).

\section{CONCLUSIONES}

Se ha diseñado y validado por primera vez en España un instrumento que sirve para conocer de manera sistemática los factores sociales y culturales que influyen en las experiencias sobre la menstruación. El METCON es una escala de 22 ítems compuesta por cuatro dimensiones que miden los mitos, estereotipos de género, tabúes y conocimientos, por lo que permite abordar el estudio de la menstruación de una forma integral (biológica, cultural y social).

Las jóvenes encuestadas reflejan conocimientos insuficientes sobre el ciclo menstrual y principalmente sobre la menstruación. Además, poseen conocimientos diferentes entre menstruación y ciclo menstrual, reflejando menos conocimientos con respecto a la menstruación que con respecto al ciclo menstrual. Esta falta de conocimientos está relacionada con que el tabú menstrual sigue estando presente en familias y contextos.

Es necesario romper el tabú menstrual, por lo que es fundamental que se analicen los contenidos impartidos a lo largo de la etapa escolar (Primaria, Secundaria y Bachillerato) detectando lagunas de conocimiento e implementado más formación en ambos sexos adaptada a la edad, donde se expliquen y aclaren dudas con respecto a la menstruación desde un punto de vista biológico, fisiológico y cultural. En Andalucía, el II Plan estratégico de Igualdad de Género en Educación (2016-2021), publicado en el BOJA de 2 de marzo de 2016, puede ayudar en este sentido.

La utilidad de la escala METCON (Botello-Hermosa 2018) es ayudar a detectar la existencia del tabú menstrual, mitos e ideas falsas sobre la menstruación (que puedan ser la base de embarazos no deseados en jóvenes), estereotipos de género y conocimientos; y como consecuencia, ayudar a facilitar un manejo adecuado del ciclo menstrual en consultas de Atención Primaria y/o en el entorno escolar. 
Alicia Botello-Hermosa, María García-JimÉneZ,

Nicia del Rocio SANTANA-BERlanga y CECILIA Ruiz-FERRón

Diseño y validación de un instrumento para medir los conocimientos y actitudes de las mujeres jóvenes ante la menstruación: escala Metcon (Botello-Hermosa 2018)

\section{REFERENCIAS BIBLIOGRÁFICAS}

Barr, Elissa M., Michelle J. Moore, Tammie Johnson, Jamie Forrest y Melissa Jordan. «New evidence: data documenting parental support for earlier sexuality education». Journal of School Health 84 (2014): 10-17.

Boletín Oficial del Estado. Instrumento de ratificación del Convenio del Consejo de Europa sobre prevención y lucha contra la violencia contra la mujer y la violencia doméstica, 11 de mayo 2011. BOE núm. 137 de 6 de junio de 2014.

Botello-Hermosa, Alicia. Aproximación a las creencias populares sobre los ciclos vitales femeninos desde la perspectiva de género. Tesis Doctoral, U de Sevilla, 2013. http://fondosdigitales.us.es/tesis/tesis/2197/aproximacion-las-creencias-populares-sobre-los-ciclos-vitales-femeninos-desde-la-perspectiva-de-genero/. Consultado el 5 septiembre de 2018.

Botello-Hermosa, Alicia, y Rosa Casado-Mejía. «Miedos y temores relacionados con la menstruación: Estudio cualitativo desde la perspectiva de género». Texto \& Contexto Enfermagem 24.1 (2015): 13-21. http://dx.doi. org/10.1590/0104-07072015000260014

Botello-Hermosa, Alicia, y Rosa Casado-Mejía. «Estereotipos de género con respecto a las etapas reproductivas de las mujeres y sus implicaciones en la salud». Matronas profesión 4 (2016): 130-136. Recuperado de http://www. federacion-matronas.org/wp-content/uploads/2018/01/original-estereotipos-de-genero.pdf. Consultado el 15 de noviembre de 2018.

Bourdieu, Pierre. «Symbolic Violence». Revista Latina de Sociología 2 (2012): 1-4. http://oaji.net/articles/2017/4754-1517576633.pdf

Brooks-Gunn, Jeanne, y Dianne N. Ruble. «The menstrual attitude questionnaire». Psychosomatic Medicine 42 (1980): 503-12.

Cronbach, Lee Joseph. "Coefficient alpha and the internal structure of tests». Psychometrika 16.3 (1951): 297-334. doi: 10.1007/bf02310555

Feijóo Tituana, María Beatriz. Tapua: La menstruación como parte de los ciclos de violencia simbólica. Producto o presentación artística. Trabajo de Titulación, Universidad de San Francisco de Quito, 2016. (http://repositorio.usfq.edu. ec/bitstream/23000/5930/1/128922.pdf). Consultado el 7 septiembre 2018.

Firat, Mehmet, Özen Kulakaç, Selma Öncel y Arzu Akcan. «Menstrual Attitude Questionnaire: confirmatory and exploratory factor analysis with Turkish samples». Journal of Advanced Nursing 65 (2009): 652-662. doi: 10.1111/j.13652648.2008.04919.x Consultado el 25 enero de 2019.

Feminismo/s 33, junio 2019, pp. 225-247 
Alicia Botello-Hermosa, María García-JimÉneZ,

Nicia del Rocio SANTANA-BERlanga y CECILIA Ruiz-FERRón

Diseño y validación de un instrumento para medir los conocimientos y actitudes de las mujeres jóvenes ante la menstruación: escala Metcon (Botello-Hermosa 2018)

Goel, Manish Kumar y Kundan Mittal. «Psycho-social behaviour of urban Indian adolescent girls during menstruation». Australian Medical Journal 4.1 (2011): 48-52. doi: http//dx.doi.org/10.4066/AMJ.2011.534

Iglesia-Benavides, José Luis. «La Menstruación: un asunto sobre la Luna, venenos y flores». Medicina Universitaria 11 (2009): 279-87.

Larrañaga, Isabel, Unai Martín y Amaia Bacigalupe. «Salud sexual y reproductiva, y crisis económica en España. Informe SESPAS 2014». Gaceta Sanitaria 28. S1 (2014): 109-115. http://gacetasanitaria.org/ es-salud-sexual-reproductiva-crisis-economica-articulo-S0213911114000880

McClive, Cathy. Menstruation and Procreation in Early Modern France. Routledge: New York, 2016.

Martínez San Andrés, Francisca, Nuria Parera Junyent y Joan Rius Tarruella. «Características e impacto de la menstruación en la mujer española: el porqué del interés de la posibilidad de supresión de la menstruación». Medicina Reproductiva y Embriología Clínica 5 (2018): 71-9. DOI: 10.1016/j. medre.2017.10.002

Marván, María Luisa, Sandra Cortés-Iniestra y Regina González. «Beliefs About and Attitudes Toward Menstruation Among Young and Middle-Aged Mexicans». Sex Roles 53.3-4 (2005): 273-279. doi:10.1007/s11199-005-5685-3

Newton, Victoria Louise. Everyday Discourses of Menstruation: Cultural and Social Perspectives. London: Palgrave McMillan, 2016.

Osterlind, Steven J. Constructing test items. Boston: Kluwer, 1989.

Orringer, Kelly y Sheila Gahagan. «Adolescent Girls Define Menstruation: A Multiethnic Exploratory Study». Health Care For Women International 31.9 (2010): 831-847. doi: 10.1080/07399331003653782

Parera, Nuria, y Clara Colomé. «Menstruación en adolescentes: ¿qué podemos esperar?». Anales de Pediatría Continuada 8.6 (2010): 271-8.

Rus García, Amelia. «Óvulos rojizos». Gaceta Sanitaria 23 (2009): 256.

Sau, Victoria. Reflexiones feministas para principios de siglo. Madrid: Horas y horas, 2000.

Stein, Elissa, y Susan Kim. Flow: The cultural study of menstruation. New York: St. Martin's Griffin, 2009.

Valls-Llobet, Carme. Mujeres, salud y poder. 2. ${ }^{a}$ ed. Madrid: Editorial Cátedra, 2010. 